\title{
AN ECONOMIC MODEL OF SCIENTIFIC RULES
}

\section{José Luis Ferreira*}

Universidad Carlos III de Madrid

Jesús Zamora-BoniLla ${ }^{\dagger}$

Universidad Nacional de Educación a Distancia

Empirical reports on scientific competition show that scientists can be depicted as self-interested, strategically behaving agents. Nevertheless, we argue that recognition-seeking scientists will have an interest in establishing methodological norms which tend to select theories of a high epistemic value, and that these norms will be still more stringent if the epistemic value of theories appears in the utility function of scientists, either directly or instrumentally.

\section{INTRODUCTION}

Although some aspects of scientific research have been thoroughly studied from an economic point of view, little effort has been made until now to illuminate, with the tools of contemporary microeconomics, one of the most fundamental elements of the research process: the norms that determine what hypothesis must be taken as the right solution to each scientific problem. The problem of how to establish these methodological norms has traditionally been considered as a topic for methodologists, epistemologists or statisticians. Nevertheless, it is widely agreed that there is no algorithm allowing to prove the truth or the falsity (not to mention the relevance, or the verisimilitude) of any scientific theory under any feasible

* The author gratefully acknowledges financial support from DGI grant BEC2002-03715 (Ministerio de Educación y Cultura).

$\dagger$ The author gratefully acknowledges financial support from grants PB98-0495-C08-01 and BFF2002-03656 (Ministerio de Educación y Cultura). 
corpus of empirical evidence. Hence, the acceptance as certified knowledge of a theory, model or datum by the members of a scientific community is to a great extent a conventional decision, and hence, it always calls for some collective criteria. We propose to study the choice of these criteria under the assumption that the scientists who make this choice are in fact rational agents, not less rational than consumers, entrepreneurs or politicians as they are depicted by traditional economic models.

Some of the authors who have explicitly taken the emergence of methodological practices as a legitimate explanandum for social science have been those included in the so-called sociology of scientific knowledge and constructivist programs. Nevertheless, in the first program (see, e.g. Bloor 1976) scientists are seen as people driven by their class interests, rather than by rational choice. In turn, the second program (see Latour 1987; Knorr-Cetina 1981) rightly considered scientists' behavior as guided by the pursuit of their personal interests, basically the search for recognition among their peers. However, these authors derived from this conception the idea that what is taken as certified knowledge is just the result of the work of those scientists who have been more successful in assembling resources and allies in favor of their views and projects. Neither of these approaches leaves a relevant place for the working of methodological norms as constraints in the process of theory choice, save as mere rhetorical devices, having nothing to do with the objective value of knowledge. Of course, these authors, being mostly sociologists and anthropologists, have never tried to illuminate their discussions by means of economic or game theoretical modeling. To our knowledge, the only two exceptions where an economic analysis has been made of the epistemic properties of some systems of scientific norms are Goldman and Shaked (1991) and Kitcher (1993) but, in these models, the norms are imposed on the scientific community "from above", whereas in ours we will analyze those norms which researchers would prefer to impose on themselves. Another interesting economic model of some epistemic aspects of scientific research is Brock and Durlauf (1999), but it deals with the choice of theories, rather than with the choice of scientific standards.

In this paper we basically try to show the following: first, how the pursuit of recognition by rational scientists can be an explanatory factor for the choice of specific methodological rules; second, that the rules so chosen will usually lead researchers to accept theories of high quality; and third, that some common features of the rules actually used in science show that researchers must also have an interest in the quality of the accepted theories, besides an interest in recognition. Our general notion of a methodological rule is that of a set of tests (that the proposed solutions to a given scientific problem might pass or fail to pass), together with a specification of what, or at least how many, of those tests an attempted solution should pass in order to be taken as an acceptable solution. Stated 
differently: a methodological rule can be seen as the combination of a scale (measuring, or at least ordering the possible epistemic values a theory may have, according to what tests it has overcome), and a point (or points) on the scale separating acceptable theories from unacceptable ones. We cannot offer an explanation of what tests are considered as relevant by scientists in the first place, or of the rules that tell how are tests appropriately performed. The answer to these questions will depend on the details of the knowledge accumulated in each scientific discipline (although we do not disregard the fact that some relevant rational-choice arguments could be proposed in order to explain some general features of the choice of tests). So, our objective in this paper will be the second element of methodological rules: why acceptable theories must pass a specific number or combination of tests.

Before entering into the details of our model, let us introduce, as stylized facts, some general features of the working of actual methodological rules, that will help us in the ensuing discussions:

(1) In empirical science, no methodological rule can prove the absolute truth of any theory or hypothesis; it is not only that new tests can always disprove the accepted theories, but rather that most scientific statements are usually known to be false (they may contain idealizations, simplifications, references to fictional entities, or even empirical anomalies, i.e. known false predictions). ${ }^{1}$ In spite of this, many statements are taken as acceptable solutions to scientific problems.

(2) Acceptability is itself an ambiguous idea: a scientific statement can be acceptable either in the sense that the members of a discipline take it as the right solution to some problem, or in the sense that they take it as worth being used as if it were right. In the first sense, the discipline commands acceptance of the statement, whereas, in the second sense, it just allows acceptance of it, often allowing simultaneously the acceptance of different solutions. So, we not only find agreement and dissent in scientific disciplines in varied proportions, but also cases of consensuated dissent. ${ }^{2}$

(3) Notwithstanding the difference just indicated, for many scientific problems often no acceptable solution (even in the weakest sense) is actually discovered, and so these problems count as unsolved for the relevant scientific community.

(4) Mature disciplines are characterized by the existence of a relatively high consensus on how to determine (qualitatively, at the least) the value of each proposed solution; this is one of the aspects of normal

1 Two classical references are Popper (1959), and Cartwright (1983).

2 See, e.g. Cole (1992). 
science, according to Kuhn. Revolutions, on the other hand, are characterized basically by the transformation of that consensus into a new one. ${ }^{3}$

The approach that will be pursued in this paper is a contractarian one. By this we mean that methodological rules are seen as the result of an agreement about the circumstances under which a scientific statement becomes acceptable within a discipline (in either of the two senses mentioned above). In this respect, our approach differs from those that describe scientific order as the emergent outcome of some market-like mechanism (see, e.g. Polany 1962; Radnitzky 1987; Hull 1988; for arguments against the metaphor of science as a free market see Callon 1994; Wible 1998; Mäki 1999). We will try to show, instead, how researchers interested in their own reputation, and perhaps in other epistemic goals, will necessarily have a preference for the establishment of certain methodological rules over others, and hence, that a plausible explanation of the existence of certain rules is that they have been chosen by scientists according to this preference. In our simplified model, this choice is made under a veil of ignorance, i.e. before knowing what hypothesis is going to be developed by each researcher, and even before knowing what problems those hypotheses will have to solve. This justifies two important assumptions of our model: first, the expectation of success is a priori the same for all researchers (or, at least, the rules will be chosen according to the expectation of success of the average scientist); second, a consensus exists on how to assess the scientific value of every hypothesis, according to the tests each one has passed (since under the veil of ignorance the specific nature of these tests is ignored, the epistemic value of each hypothesis can be identified with the number of overcome tests, perhaps weighting each test by the expectation of finding a theory which passes it). Regarding this assumption, we recognize that the relative importance that has to be attached to each test is often a hotly disputed question within scientific controversies; but such disputations take place once the specific tests (and usually several theories) are known, and refer, then, to the question of where each theory must be located on the scale of epistemic values; the disputation presupposes that somewhere on the scale there is already a threshold dividing acceptable from unacceptable theories. We can draw an analogy with the case of law: the choice of a methodological rule is comparable to the enactment of a law by a legislature, whereas the disputations about the value of each test are more similar to trials, where preexisting laws have to be applied. ${ }^{4}$

${ }^{3}$ See Kuhn (1970), and chapter 13 in Kuhn (1977).

${ }^{4}$ Other arguments regarding the pertinence of giving a separate explanation of the choice of an epistemic value scale are given in Zamora-Bonilla (2002), which also presents an 
The structure of the paper is the following. Section 2 presents the fundamental elements of some possible inference rules. Section 3 analyses the quality levels which would be optimal for researchers under the rules described in Section 2. In Section 4 we extend the model by introducing the assumption that researchers may not only have a preference for their expected levels of recognition, but also for the epistemic quality levels per se. In Section 5 we study some aspects of the collective choice of an inference rule. In Section 6 we drop the assumption of preferences for the epistemic value. Section 7 presents some concluding comments. Finally, an appendix contains the proofs of the propositions.

\section{THE MODEL}

Let $N=\{1,2, \ldots, n\}$ be a finite population of scientists. Scientist $i \in N$ will develop a theory with epistemic value $x^{i}$. The epistemic values of the different theories are assumed to be i.i.d. random variables defined on $\Re_{+}$, with density function $f(x)$. The probability that the epistemic value $x^{i}$ is no greater than a threshold $x$ is given by the distribution function $F(x)=$ $\int_{0}^{x} f(x) d x$. Denote by $\bar{x}$ the maximum epistemic value attainable by any theory, i.e. the smallest value of $x$ such that the probability of developing a theory of quality superior to $x$ is zero; or, formally, $\bar{x}=\inf \{x \mid F(x)=1\}$. If $F(x)$ goes asymptotically to one, then $\bar{x}=\infty$.

The scientific population $N$ will select among the theories according to an inference rule. Let $T=\left\{x^{1}, x^{2}, \ldots, x^{n}\right\}$ be the set of theories (represented by their epistemic values) developed by the scientists in N. For simplicity, we assume that each scientist devises just one theory, and that all the devised theories are different. For simplicity we consider that $f(x)$ is such that the probability of two theories having the same epistemic value is zero. An inference rule is a choice operator of the form $I R(T) \subset T$. We consider three possible types of inference rules. Specific rules of each type will correspond to different choices of a minimum standard.

(i) $\operatorname{IR}_{1}(T)=\left\{x^{i} \mid x^{i}=\max _{j \in N}\left\{x^{j}\right\}, x^{i} \geq x\right\}$

(ii) $I R_{2}(T)=\left\{x^{i} \mid x^{i} \geq x\right\}$ and $\left.\forall j \neq i, x^{j}<x\right\}$

(iii) $\quad \operatorname{IR}_{3}(T)=\left\{x^{i} \mid x^{i} \geq x\right\}$

In words, $I R_{1}$ means that a theory is accepted if no other theory has a greater epistemic value and if it satisfies a minimum standard $x$. Inference

informal version of some of the models deployed here. On the other hand, philosophers of science have developed several "rational reconstructions" of scientists' epistemic preferences (actual or ideal), which might be employed in this paper as interpretations of our (uninterpreted) notion of epistemic value (see, e.g. Gillies 2000). In particular, Popper's idea of verisimilitude as the epistemic goal of science has led to an interesting research program on this philosophical problem. See, e.g. Zamora Bonilla (1999). 
rule $I R_{2}$, however requires that the theory is the only one that satisfies this minimum. Finally, according to $I R_{3}$, all theories that pass the minimum are selected. The difference between $I R_{1}$ and $I R_{2}$, on the one hand, and $I R_{3}$, on the other hand, may roughly correspond to the two different concepts of acceptability indicated in Section 1 (see stylized fact number 2). There are arguments to defend each rule, but they are better discussed after knowing some of the implications of adopting them, as seen in the next section.

Scientist $i$ has a utility function of the form

$$
u^{i}\left(x^{i}\right)= \begin{cases}u & \text { if }\left\{x^{i}\right\}=I R(T) \\ v & \text { if }\left\{x^{i}, x^{j}\right\} \subset I R(T) \text { for some } j \neq i \\ 0 & \text { if } x^{i} \notin \operatorname{IR}(T),\end{cases}
$$

with $u \geq v \geq 0$ and $u>0$.

The assumption that the epistemic values of the different theories are assumed to be i.i.d. random variables deserves some discussion. Different theories may propose different models to explain (approximately) the same set of phenomena. Also, different theories may propose different models to explain different (but not totally) sets of phenomena. In either situation, it is not necessarily the case that one theory is right and the other is wrong, even if they contradict each other; e.g. they may be particular cases of a more encompassing theory yet to be discovered. Furthermore, it may happen that two theories are logically equivalent, but that one of them is more valuable (e.g. is simpler or provides a better intuition). Thus, a high epistemic value of one theory does not necessarily imply that the epistemic value of any other is low. One theory may have some virtues, but an alternative theory may also have other virtues. This way, the statistical correlation of the quality of different theories may be hard to establish in advance, which justifies our assumption that the epistemic values are i.i.d.

To put it in another way, if the epistemical value is identified with its usefulness for solving problems, one simple way to define $x$ is to make it represent the number of problems that the theory may solve according to the scientific community. Clearly, two different theories may solve the same number of problems even if they are not fully compatible.

$I R_{1}, I R_{2}$ and $I R_{3}$ represent three different types of inference rules. Our work focuses on the choice of a rule within each type. We do not attempt to provide a theory about when and why a type of inference rule is chosen. We use the three types because they all resemble the way theories are actually selected. Depending on the particular problem and the actual stage of development of a given science, one type may be preferred to another. In our model this will represent what it means for the members of a scientific community to have a solution to a problem. What our model allows for is discussion of the value that any specific methodological rule may have for those scientists. 


\section{OPTIMAL INFERENCE RULES}

The inference rules defined above are characterized by a parameter $x$. For each rule the expected utility of a scientist depends on the value of $x$. It will then be of interest to find the value that maximizes his expected utility.

Proposition 1. Under the inference rule $I R_{1}$, scientists' maximum expected utility is $\frac{u}{n}$, attained at $x_{1}^{*}=0$.

The reason why the threshold $x_{1}^{*}=0$ is simple. Each scientist receives a greater utility if his theory is accepted. With $x_{1}^{*}=0$, all theories pass the threshold, and then the probability of acceptance is $\frac{1}{n}$. If the threshold $x$ increases, there is a positive probability that each scientist's own theory does not pass the threshold, thus reducing the probability of being selected. Notice also that $x_{1}^{*}=0$ does not mean that 'anything goes' as a theory, since the rule selects the theory with the highest value. As a consequence, this rule has an interesting property, namely, that there is always just one solution to every problem.

The expected epistemic value of the theory chosen under $I R_{1}$ (denoted by $\left.E\left(I R_{1}\right)\right)$ is

$$
\begin{aligned}
E\left(I R_{1}\right) & =E\left(\max _{i \in N}\left\{x^{i}\right\}\right) \\
& =\int_{0}^{\infty} n F(x)^{n-1} x d x .
\end{aligned}
$$

Where $n F(x)^{n-1}$ is the density function of $F(x)^{n}$, the distribution function of $\max _{i \in N}\left\{x^{i}\right\}$.

Proposition 2. Under the inference rule $I R_{2}$, the threshold that maximizes scientists' expected utility is $x_{2}^{*}$ that satisfies $\bar{x}>x_{2}^{*}>0$ and $F\left(x_{2}^{*}\right)=1-\frac{1}{n}$, giving an expected utility of $\frac{u}{n} F\left(x_{2}^{*}\right)^{n-1}$.

Corollary 1. $x_{2}^{*} \rightarrow \bar{x}$ as $n \rightarrow \infty$.

To gain insight as to why $\bar{x}>x_{2}^{*}>0$ see that if either $x_{2}^{*}=0$ or $x_{2}^{*} \geq \bar{x}$, then for each scientist the probability of having his own theory accepted is zero (in the first case, because all theories pass the threshold, and, in the second case, because no one passes it). Hence, a positive (but not too high) threshold is necessary to maximize the utility. According to this rule, the probability of having a solution for a given problem is

$$
\begin{aligned}
n(1-F(x)) F(x)^{n-1} & =n \frac{1}{n}\left(1-\frac{1}{n}\right)^{n-1} \\
& =\left(1-\frac{1}{n}\right)^{n-1} .
\end{aligned}
$$


Observe that, if $n=2$, this probability has a value of 0.5 , and that it converges monotonically to $\lim _{n \rightarrow \infty}\left(1-\frac{1}{n}\right)^{n-1}=e^{-1}$. i.e. the probability of obtaining a solution decreases with the number of scientists down to the limit $e^{-1}$.

We can now compute the expected epistemic value of the theory chosen under $I R_{2}$. Since there is a positive probability that no theory is chosen, we have at least two options to calculate the expected epistemic value. Denote by $E\left(I R_{2} \mid I R_{2}=\emptyset\right)$ the expected epistemic value conditional upon obtaining a solution, and by $E\left(I R_{2}\right)$ the unconditional expected epistemic value, where the event in which no theory is chosen is identified with having a theory with zero quality.

Propositions 1 and 2 imply that the minimum standard is greater under $I R_{2}$ than under $I R_{1}\left(x_{2}^{*}>x_{1}^{*}\right)$. However, it is interesting to note that the expected epistemic value under $I R_{2}$ may be lower or higher than under $I R_{1}$. Nevertheless, our simulations show that, for reasonable distribution functions, $E\left(I R_{2} \mid I R_{2} \neq \emptyset\right)>E\left(I R_{1}\right)$ whereas $E\left(I R_{2}\right)<E\left(I R_{1}\right)$. This is true if, for instance, $x$ follows a uniform distribution. In the Appendix, Example 1 shows the details.

Proposition 3. Under the inference rule $I R_{3}$, if $v$ is sufficiently smaller than $u$, then the epistemic value $x_{3}^{*}>0$ that maximizes scientists' expected utility solves $(n-1) F(x)^{n-2}-n F(x)^{n-1}=\frac{v}{u-v}$. If $v$ is sufficiently close to $u$, scientists' expected utility is maximized at $x_{3}^{*}=0$.

Corollary 2. $x_{2}^{*} \geq x_{3}^{*}$.

Obviously, the probability of having a solution under $I R_{3}$ is higher than under $I R_{2}$ and lower than under $I R_{1}$ (although the expected number of solutions under $I R_{3}$ is typically larger than under $\left.I R_{1}\right)$. The optimal utility level under $I R_{1}$ is higher than under $I R^{2}\left(\frac{u}{n}>\frac{u}{n} F\left(x_{2}^{*}\right)^{n-1}\right)$. When $u$ $=v$, the optimal threshold under $I R_{3}$ is $x_{3}^{*}=0$, with $E u_{3}(0)=u>\frac{u}{n}$, while if $v=0, I R_{3}$ coincides with rule $I R_{2}$, and $x_{3}^{*}=x_{2}^{*}$, with $E u_{3}\left(x_{3}^{*}\right)=E u_{2}\left(x_{2}^{*}\right)=$ $\frac{u}{n} F(x)^{n-1}$. In any case, $x_{2}^{*}$ is never lower than either $x_{1}^{*}$ or $x_{3}^{*}$.

Tables 1 and 2 below summarize our findings.

\begin{tabular}{|c|c|c|c|c|c|c|}
\cline { 3 - 7 } \multicolumn{2}{c|}{} & \multicolumn{5}{c|}{$I R_{3}$} \\
\cline { 2 - 7 } \multicolumn{1}{c|}{} & $I R_{1}$ & $I R_{2}$ & $v=u$ & $v \rightarrow u$ & $v \rightarrow 0$ & $v=0$ \\
\hline$x_{1}^{*}$ & 0 & $x_{2}^{*}>0$ & 0 & 0 & $0<x_{3}^{*}<x_{2}^{*}$ & $x_{3}^{*}=x_{2}^{*}$ \\
\hline$E u_{\mathrm{i}}$ & $\frac{u}{n}$ & $\frac{u}{n}\left(1-\frac{1}{n}\right)^{n-1}$ & $u$ & $v$ & $E u_{2}<E u_{3}<v$ & $E u_{3}=E u_{2}$ \\
\hline
\end{tabular}




\begin{tabular}{|c|c|}
\hline$x_{2}^{*}>x_{1}^{*}$ & $x_{2}^{*} \geq x_{3}^{*}$ \\
\hline$E u_{2}<E u_{1}$ & $E u_{2} \leq E u_{3}$ \\
\hline
\end{tabular}

TABLE 2

The facts that $x_{2}^{*}>x_{1}^{*}$ and $E\left(I R_{2} \mid I R_{2} \neq \emptyset\right)>E\left(I R_{1}\right)$ may be interesting social arguments in favor of rule $I R_{2}$ rather than $I R_{1}$ in some situations. Obviously, all else being equal, a higher minimum standard is socially preferred. Whether the inequality $E\left(I R_{2} \mid I R_{2} \neq \emptyset\right)>E\left(I R_{1}\right)$ presents a social argument in favor of rule $I R_{2}$ depends on the attitude of scientists. If $I R_{2} \neq \varnothing$ implies that the scientific community will continue producing theories for the problem at hand for one more period, and so on, until $I R_{2} \neq \emptyset$, then, eventually, the expected epistemic value under $I R_{2}$ will be greater than under $I R_{1}$. On the other hand, if $I R_{2} \neq \emptyset$ implies that the search for a theory is abandoned, then the fact that $E\left(I R_{2}\right)<E\left(I R_{1}\right)$ makes $I R_{1}$ more attractive socially (but then this must be balanced with the other fact that $x_{2}^{*}>x_{1}^{*}$.)

The differences between rules $I R_{1}$ and $I R_{2}$ correspond to different degrees of a conservative attitude. Under rule $I R_{2}$, the judgment is suspended if there is more than one theory satisfying the minimum requirements, and so, fewer scientific problems are solved and scientists receive recognition less frequently. Hence, we can expect that scientists who are fundamentally motivated by the pursuit of recognition will tend to prefer $I R_{1}$, and hence a null threshold (at least if they want to have some theories accepted in the strong sense, for in general $I R_{3}$ only allows them to consider theories acceptable in the weak sense). The problem is, of course, that the stylized fact number three of the scientific method described at the end of Section 1 seems to entail that scientists do establish non-zero thresholds. We suggest that this result should be interpreted as a falsification of the assumption that scientists only care about recognition, i.e. their utility function must contain some additional elements that make it reasonable for them to establish a positive threshold for acceptable theories. We explore this possibility in the next sections.

Before beginning this discussion, we want to notice that our inference rules have both a family resemblance with the method of eliminative induction, as well as significant differences with it. This method consists in displaying all the conceivable solutions to a problem, and testing them empirically until all of them, save probably one, become falsified (if all are contradicted by the data, then some presupposition in the construction of the hypotheses must be false.) Our rules, instead, establish ex ante a threshold that acceptable theories must surpass, and it is only ex post that it is known how many hypotheses happen to survive the proof. Although it has been argued that eliminative induction is regularly used 
by empirical scientists (see, e.g. Kitcher 1993), there are strong conceptual arguments showing that, in fact, it can only work under extremely restricted circumstances: first, in general not all conceivable hypotheses are actually conceived; second, usually many falsified theories can be given a second chance by making some ad hoc modifications to their assumptions; and third, as we saw in Section 1, in many cases theories are taken as acceptable even if they fail to solve all the relevant problems (or, more strongly, even if they are known to be false strictly speaking). Our rules, on the contrary, do not suffer from these problems, for they only demand that scientists take into account the probability they have of devising a good enough theory, and not the probability each conceivable theory has of being true. Our thesis is, then, that what is actually employed in scientific research is something similar to the inference rules described in this section, not eliminative induction properly understood, and that it has been the similarity between both types of norms which has led some philosophers to think that eliminative induction was the rule scientists employ.

\section{PREFERENCES FOR THE EPISTEMIC VALUE}

In this section we study the consequences on the optimal thresholds in the inference rules examined above in the case scientists also care about the epistemic value of the theories. There are, at least, two different reasonable ways of introducing this kind of preferences. In one of them, a scientist may derive more utility if his own theory has a higher epistemic value, assuming the theory is accepted. Alternatively, in a second way of modeling, preferences may have as an argument the expected quality of the chosen theory. We favor this second approach. However, the problem becomes very complicated in this case. In order to overcome this difficulty, we propose a third way, namely, to introduce the value of the threshold as an argument of the utility function. We see this way of modeling as a compromise between what should be the case and tractability. Notice that, by adopting it, we get that the higher the threshold, the higher the expected epistemic value of the accepted theory, which is what we wanted.

Assume, then, that utility levels are differentiale functions $u(x)$, and $v(x)$ with $u(0)=u, v(0)=v, u^{\prime}>0$, and $v^{\prime}>0$. Notice that if $u^{\prime}$ and $v^{\prime}$ are very small for all $x, u(x)$ and $v(x)$ can be made arbitrarily close to $u$ and $v$, respectively. Now we can state and prove the counterparts of propositions $1-3$ in the previous section. These new propositions are all about interior solutions to the maximization problem where the second order conditions for a maximum are satisfied. The comparison of corner solutions $(x=0$ or $x \geq \bar{x})$ is of limited interest.

Proposition 4. Under the inference rule $I R_{1}$, if $u(x)$ is as described above, scientists' expected utility is maximized at $\hat{x}_{1}>x_{1}^{*}=0$. 
In Proposition 1 we saw that a low threshold increased the probability of a given theory being accepted, thus increasing the utility of the scientists. Now the low threshold also implies a smaller utility because of the new preferences. The value $\hat{x}_{1}$ is the result of these two opposite forces.

An interesting normative consequence is the following. Recall (see the proof in the Appendix) that, under inference rule $I R_{1}$, the expected utility of a scientist if the epistemic value is $x$ is given by $E \hat{u}_{1}(x)=\frac{u(x)}{n}\left(1-F(x)^{n}\right)$. Suppose now that the threshold $x$ for rule $I R_{1}$ is chosen by a "science manager" with the objective of maximizing some public interest regarding the standards of accepted theories. In particular, suppose that this manager gets a utility of $w(x)>0$, with $w^{\prime}(x)>0$, if there is a theory that passes the minimum quality $x$, and zero otherwise, then she will maximize a function of the form $U(x)=w(x)\left(1-F(x)^{n}\right)$. Therefore, if $w(x)$ is proportional to $u(x)$, the manager's problem will have the same solution as the problem in Proposition 4 . This has a nice normative interpretation. If scientists are left alone, they will choose the same standards as the public (or the "science manager" who represents the public's interests in science), as long as they value equally (proportionally) the quality of knowledge.

Proposition 5. Under the inference rule $I R_{2}$, if $u(x)$ is as described above, scientists' expected utility is maximized at $\hat{x}_{2}>x_{2}^{*}$.

The intuition behind this proposition is similar to that of Proposition 4. As in the previous section, the utility level under $I R_{1}$ is greater than under $I R_{2}$. For any threshold $x$, the utility in any case (having his own theory selected or not) is the same, but the probability of having the theory accepted is more favorable under $I R_{1}$. It is interesting to note that we have been able to establish $\hat{x}_{1}>x_{1}^{*}$ and $\hat{x}_{2}>x_{2}^{*}$ regardless of $u(x)$ and $u$.

In general, we cannot establish a relationship between $\hat{x}_{1}$ and $\hat{x}_{2}$, as shown in Example 2 of the Appendix.

Notice also that expected utilities are greater when scientists have preferences for the epistemic value of the theory under either $I R_{1}$ or $I R_{2}$. For instance, under $I R_{1}$ the utility level of $\frac{u}{n}$ (the maximum if $u(x)=u$ ) can be attained if $u(x) \geq u$ just by setting $x=0$. But since the maximum is attained at $\hat{x}_{1} \neq 0$, this means that the utility must be greater in this case. The same argument can be made for $I R_{2}$.

Under inference rule $I R_{3}$, we need a stronger condition to reach a higher threshold when adding preferences for the epistemic value of theories.

Proposition 6. Under the inference rule $I R_{3}$, if $u(x)$ and $v(x)$ are as described above, if $\frac{v(x)}{u(x)-v(x)} \leq \frac{u}{u-v}$ for all $x$, and if both equations (2) and (6) in the Appendix have only one solution for a maximum, then scientists' expected utility is maximized at $\hat{x}_{3} \geq x_{3}^{*}$. 
By the same reasons mentioned earlier, we must have $E \hat{u}_{3}\left(\hat{x}_{3}\right) \geq$ $E u_{3}\left(x_{3}^{*}\right)$. To understand why we need the additional condition $\frac{v(x)}{u(x)-v(x)} \leq \frac{v}{u-v}$ to have $\hat{x}_{3} \geq x_{3}^{*}$ recall that, when $u(x)=u$ and $v(x)=v, x_{3}^{*}$ was positive or zero depending on whether $v$ was close to zero or to $u$. Now, even if $v(0)$ is close to zero and not to $u(0)$, it may occur that, as $x$ increases $v(x)$ becomes closer to $u(x)$ than to zero. This may make $\hat{x}_{3}=0$ even if $x_{3}^{*}>0$. It is to avoid this situation that we impose the new condition.

Consider the cases analyzed in Section 3, where scientists had no preferences for the quality of the selected theory, and consider also any rule that selects a theory at random from among the elements in $T$ (any rule that does not care about the epistemic value of theories and that is anonymous can be considered random). In our model, this rule will serve the same purpose as rule $I R_{1}$ from the viewpoint of the scientists (there will be one theory selected, and the expected utility for each scientist will be $\frac{u}{n}$, the same as with $I R_{1}$.) Obviously, if devising and performing tests has a positive cost for scientists, the expected utility derived from random selection will be higher than with $I R_{1}$. One nice interpretation of the results in this section is, then, that the assumption that scientists' utilities depend on the epistemic value of the chosen theory (however slight this dependence is, i.e. however close $u(x)$ is to $u$ ) provides an explanation not only of why scientists actually prefer a non-zero threshold, but also of why they are willing to perform any tests at all. A similar argument can be made for both $I R_{2}$ and $I R_{3}$.

One may criticize the introduction of the epistemic value of the threshold $x$ in the utility function on the basis that it is very close to assuming what is intended to be proven, that scientists will opt for theories with high epistemic value. However, notice that the utility of scientist $i$ is $u(x), v(x)$, or zero depending on whether his theory is the only one accepted, is accepted along with others or not accepted at all. This leaves a great deal of room for possible situations in which what matters is the strategic behavior to accept or not to accept others' theories. For example, each scientist might in principle decide to reject all the theories proposed by her colleagues, no matter how valuable they are (arguing, for example, that they are not well tested enough). Obviously, if all behaved this way, they would get utility 0 . What we have shown, so far, is that every scientist has an interest in a certain methodological norm being established, and that an optimal strategy for choosing the norm can be that of using the epistemic value thresholds as a way of selecting among theories.

The next section analyzes how this optimal value can actually be chosen strategically by scientists. In Section 6 we drop the assumption of preferences for the epistemic value and attach a practical value to better theories as an alternative model. 


\section{THE CHOICE OF THE INFERENCE RULE}

For a given choice rule, we have detected the optimal threshold under two scenarios. This does not mean that the scientific population will immediately choose that value as the minimum epistemic value for a theory to be acceptable. In fact, it cannot be even taken for granted that the current members of the scientific community are always the ones who make the choice. For example, they may have inherited certain inference rules from their predecessors. In this section we show, however, that any reasonable mechanism in which the set of decision makers is indeed the scientific population has the optimal value as an equilibrium.

Assume, then, that the inference rule is fixed, that scientists select the threshold value under a mechanism in which each scientist proposes a value $y^{i} \geq 0$, and that the value chosen by the mechanism is $x=f\left(y^{1}, \ldots, y^{n}\right)$. Next are two interesting properties that the function $f$ may satisfy. Let $y^{-i}=\left(y^{1}, \ldots, y^{i-1}, y^{i+1}, \ldots y^{n}\right)$ and $\left(y^{-i}, \tilde{y}^{i}\right)=\left(y^{i}, \ldots, y^{i-1}, \tilde{y}^{i}, y^{i+1}, \ldots y^{n}\right)$.

(i) Unanimity $(U)$ : if $y^{1}=\ldots=y^{n}=y$, then $f\left(y^{1}, \ldots, y^{n}\right)=y$, and

(ii) Influence of direction (IOD): for all $y^{-i}$ and $x$, if $\left|y^{i}-x\right|<\left|\hat{y}^{i}-x\right|$, then $\left|f\left(y^{-i}, y^{i}\right)-x\right|<\left|f\left(y^{-i}, \hat{y}^{i}\right)-x\right|$ if the sign of $f\left(y^{-i}, y^{i}\right)-x$ is the same as the sign of $f\left(y^{-i}, \hat{y}^{i}\right)-x$.

The meaning of unanimity is obvious: if all scientists propose the same threshold $y$, then that threshold is adopted. Influence of Direction means that, if the threshold proposed by scientist $i$ (say $\hat{y}^{i}$ ) is higher (alt. lower) than a given epistemic value $x$ and if an alternative threshold (say $y^{i}$ ) is even higher (alt. lower), then the value chosen by $f$ must also be higher (alt. lower) when $y^{i}$ is proposed than when $\hat{y}^{i}$ is proposed regardless of the values proposed by the other scientists.

Proposition 7 states that, under $U$, the optimal threshold is obtained in equilibrium, and Proposition 8 states that, if $I O D$ is added, then this is essentially the only equilibrium. The reason is that if the equilibrium is not $x_{i}$, by IOD, there will be a scientist who can influence an outcome still closer to $x_{i}$ and be better off.

Proposition 7. Under the mechanism described above, if $f$ satisfies $U$, then $\left(y^{1}, \ldots, y^{n}\right)=\left(x_{i}^{*}, \ldots, x_{i}^{*}\right)$ is a Nash equilibrium under rule $I R_{i}$, with $u$ and $v$ constant, and $\left(y^{1}, \ldots, y^{n}\right)=\left(\hat{x}_{i}, \ldots, \hat{x}_{i}\right)$ is an equilibrium under $I R_{i}$, with $u=$ $u(x)$ and $v=v(x)$.

Proposition 8. If, in addition, $f$ satisfies IOD, then, under rule $I R_{i}$ the only equilibrium outcome is the optimal epistemic value or $x>\bar{x}$.

Let $x_{i}$ be the optimal threshold under rule $I R_{i},\left(x_{i}=x_{i}^{*}\right.$ or $\hat{x}_{i}$, depending on whether $u$ and $v$ are constant or not) then, when we require $U$ and IOD, the only equilibria imply $f\left(y^{1}, \ldots, y^{n}\right)=x_{i}$ or $f\left(y^{1}, \ldots, y^{n}\right)>\bar{x}$. See that in 
the second case the required epistemic value is too high (recall that $\bar{x}$ is the minimum epistemic value such that $F(x)=1$ ). I.e. the epistemic value is either optimal or too high, which contradicts the extreme view in sociology of science that suggests that the individualism of scientists does not imply an optimal level, but rather, a too low epistemic value of theories.

There are at least two other ways of obtaining the same result. One is to use a mechanism in which the threshold is decided by majority rule and where Nash equilibria in (weakly) dominated strategies are ruled out. If we allow for equilibria in dominated strategies, multiple equilibria emerge, e.g. an equilibrium in which every scientist proposes the same arbitrary threshold, as, in this case, unilateral deviations will not affect the outcome of the majority rule. When weakly dominated strategies are not considered, it is in the scientists' interest to propose the best ex-ante threshold, as calculated in the different cases.

The other way is to select $x^{*}$ as the outcome of a strong Nash equilibrium. A strategy profile is not a strong Nash equilibrium if a coalition of players can simultaneously deviate and improve their utility. According to this definition, a collective deviation would take place any time a threshold $\tilde{x}_{i} \neq x_{i}$ is chosen. The concept of strong Nash equilibrium was introduced in Aumann (1959). It has been shown to be a very strong condition for an equilibrium to be immune against coalitional deviations. There are many weaker definitions of equilibria when coalitional deviations are considered, like the Coalition-proof Nash equilibrium presented in Bernheim, Peleg and Whinston (1987). However there is no need to go through this literature, as any strong Nash equilibrium also satisfies these definitions.

We have shown, then, that under reasonable conditions, and given the structure of the scientific community (number of scientists or scientists' teams, rule of inference and preferences) there will be no strategic behavior of agreement on low standards for theory choice.

\section{THEORIES WITH A PRACTICAL VALUE}

In this section we outline an alternative to the model in Section 4 that leads to the same results. Instead of assuming that scientists have direct preferences for the epistemic value we will now assume that theories may have a practical value which depends on the epistemic value. ${ }^{5}$ To this end, suppose that there is an agent who wants to use an accepted theory to guide her practical work. The theory has to have a minimum quality of, say $y$, with $y \geq x$. It is costly to look for scientific theories, so this agent will just look randomly at one of the accepted theories and, if its quality is not below $y$, will take it and pay $z$ to hire the scientist who developed

${ }^{5}$ We thank an anonymous referee for suggesting this extension. 
the theory as a consultant. If the theory has a quality below $y$, the agent abandons the search and does nothing of value for any scientist. There is no other source of utility for the scientists.

Let $P(y)$ be the cumulative probability distribution of $y$ from the scientists' point of view. I.e. $P(y)$ indicates the probability that the agent's required minimum standard is smaller than or equal to $y$. Thus, for scientist $i$ the ex-ante utility of developing a theory with value $x^{i}$ can be defined as

$$
u^{i}=u\left(x^{i}\right)=\frac{z P\left(x^{i}\right)}{k} \text { if } t^{i} \in I R_{i}(T),
$$

where $k$ is the cardinal of $I R_{i}(T)$, and

$$
u^{i}=0 \text { if } t^{i} \notin I R_{i}(T),
$$

where $P\left(x^{i}\right)=\operatorname{prob} .\left(y \leq x^{i}\right)$ indicates the probability that the theory of scientist $i$ passes the agent's required minimum standard. The accumulative function $P($.$) is increasing. If we assume further \frac{\partial P(y)}{\partial y}>0$, then we have $u^{\prime}>0$, and the counterparts of propositions $4,5,6$, and 8 follow for $I R_{1}$ and $I R_{2}$. So, the choice of a high epistemic standard may be due not only to the scientists' preferences for theories with a high epistemic value, but also to the existence of some correlation between the epistemic and the pragmatic values of theories.

\section{CONCLUSION}

We have developed an economic theory for the choice of epistemic standards. Our model assumes only selfish, strategically behaving scientists and, nevertheless, implies that theories satisfying stringent standards will tend to be accepted, especially if scientists do not seek, so to speak, the bare recognition of getting their theories accepted, but the recognition for having devised a good solution to a scientific problem (this refers to our assumption that, when their theories become accepted, scientists receive a higher utility the higher the epistemic value of their theories). The same conclusion follows if a high epistemic value is instrumental for the attainment of other practical goals, for which they may be paid. Hence, the fact that scientific standards are the outcome of a social negotiation does not entail that scientific knowledge lacks real epistemic value; on the contrary, under some reasonable assumptions, the negotiation will lead to the establishment of very demanding scientific norms. We can here draw an analogy with the case of sports: it is true that the norms regulating, say, an athletic competition are the result of a negotiation, and that athletes are basically driven by the will to win and the pursuit of glory, but we cannot infer from this that the winners 
in the competition are not, objectively, much more qualified for those competitions than the average person.

Obviously, our model does not prove that actual scientific norms are systematically efficient, for our assumptions are just idealizations attempting to capture some essential aspects of those norms, and not a full empirical description of them. But what our model suggests is that, in order to argue against the objective validity of some parts of scientific knowledge, something more is needed besides just pointing to the fact that this knowledge is the outcome of a social negotiation: at the very least, an argument should be offered showing that the scientific standards arising from that negotiation are inefficient in a clear epistemic sense, i.e. that they systematically preclude the acceptance of theories which are objectively better than the ones actually accepted.

The model, of course, can be extended in several directions. Perhaps, the most natural of them is the introduction of heterogenous scientists, with differences in the probability of developing a theory of a minimum quality, different preferences, or differences in authority within the community. The study of theses extensions is left for a future work.

\section{APPENDIX}

Proof of Proposition 1. The expected utility of a scientist if the epistemic value is $x$ is given by

$$
\begin{aligned}
E u_{1}^{*}(x) & =\int_{x}^{\infty} f(y) F(y)^{n-1} u d y \\
& =\frac{u}{n}\left[F(y)^{n}\right]_{x}^{\infty} \\
& =\frac{u}{n}\left(1-F(x)^{n}\right) .
\end{aligned}
$$

$\frac{u}{n}$.

The maximum of $1-F(x)^{n}$ is attained at $x=0$. Since $F(0)=0, E u_{1}^{*}(0)=$

Proof of Proposition 2. The expected utility of a scientist if the epistemic value is $x$ is given by

$$
\begin{aligned}
E u_{2}^{*}(x) & =(1-F(x)) F(x)^{n-1} u \\
& =\left(F(x)^{n-1}-F(x)^{n}\right) u .
\end{aligned}
$$

First order conditions for a maximum are given by

$$
\left((n-1) F(x)^{n-2}-n F(x)^{n-1}\right) f(x) u=0 .
$$

As $f(x), u>0$, this implies

$(n-1) F(x)^{n-2}-n F(x)^{n-1}=0$, 
or

(1) $F(x)=\frac{n-1}{n}=1-\frac{i}{n}$.

See that the solution of this equation gives $x_{2}^{*}>0$, unless $n=1$, since $F(0)=0$. Substituting condition (1) in the objective function one gets $E u_{2}^{*}\left(x_{2}^{*}\right)=\frac{u}{n} F\left(x_{2}^{*}\right)^{n-1}$.

Proof of Corollary 1. Straightforward.

Example 1. Showing $E\left(I R_{2} \mid I R_{2} \neq \emptyset\right)>E\left(I R_{1}\right)$ and $E\left(I R_{2}\right)<E\left(I R_{1}\right)$ : If $F(x)=x$, then $F\left(\max _{j}\left\{x^{j}\right\}\right)=x^{n}$, and $f\left(\max _{j}\left\{x^{j}\right\}\right)=n x^{n-1}$. Thus

$$
E\left(I R_{1}\right)=\int_{0}^{\infty} n x^{n-1} x d x=\frac{n}{n+1} .
$$

According to $I R_{2}$, the chosen theory is also the one with the maximum value. If this theory passes the threshold $x_{2}^{*}$, then it is distributed with uniform probability between $x_{2}^{*}$ and one if no other theory passes the threshold. By (1) $F\left(x_{2}^{*}\right)=x_{2}^{*}=1-\frac{1}{n}$. Therefore

$$
E\left(I R_{2} \mid I R_{2} \neq \emptyset\right)=\frac{1+\left(1-\frac{1}{n}\right)}{2}=1-\frac{1}{2 n} .
$$

It follows that $E\left(I R_{2} \mid I R_{2} \neq \emptyset\right)>E\left(I R_{1}\right)$ for all $n \geq 1$.

Finally, the probability of having one theory chosen under $I R_{2}$ is $\left(1-\frac{1}{n}\right)^{n-1}$, which means that

$$
E\left(I R_{2}\right)=\left(1-\frac{1}{2 n}\right)\left(1-\frac{1}{n}\right)^{n-1} .
$$

To see that $E\left(I R_{2}\right)<E\left(I R_{1}\right)$ notice that

$$
\left(1-\frac{1}{2 n}\right)\left(1-\frac{1}{n}\right)^{n-1}<\left(1-\frac{1}{n}\right)^{n-1}<1-\frac{1}{2 n}
$$

for all $n \geq 1$.

Proof of Proposition 3. The expected utility of a scientist if the epistemic value is $x$ is given by

$$
E u_{3}^{*}(x)=(1-F(x)) F(x)^{n-1} u+(1-F(x))\left(1-F(x)^{n-1}\right) v
$$

First order conditions for a maximum are given by

$$
\begin{gathered}
\left((n-1) F(x)^{n-2}-n F(x)^{n-1}\right) f(x) u+\left(n F(x)^{n-1}-(n-1) F(x)^{n-2}-1\right) f(x) v \\
\left.=\left((n-1) F(x)^{n-2}(u-v)-n F(x)^{n-1}(u-v)-v\right)\right) f(x)=0,
\end{gathered}
$$


which gives

(2) $(n-1) F(x)^{n-2}-n F(x)^{n-1}=\frac{v}{u-v}$.

Equation (2) may have multiple solutions, one being the global maximum $x_{3}^{*}>0$ if $v$ is sufficiently small. If $v$ is close to $u$, the equation has no solution, meaning that the expected utility is maximized at a corner solution, with $x_{3}^{*}=0$.

Proof of Corollary 2. Notice that Equation (2) may be rewritten as

(3) $F(x)=\frac{n-1}{n}-\frac{v}{(u-v) n F(x)^{n-2}}$.

Since $F(x)$ is non-decreasing and $\frac{v}{(u-v) n F(x)^{n-2}}>0$, the solution to Equation 2 must be strictly smaller than the solution to Equation 1 unless $v=0$, in which case $x_{2}^{*}=x_{3}^{*}$.

Proof of Proposition 4. The expected utility of a scientist if the epistemic value is $x$ is given by

$$
E \hat{u}_{1}(x)=\frac{u(x)}{n}\left(1-F(x)^{n}\right) .
$$

First order conditions for an interior maximum are given by the equation

(4) $\frac{u^{\prime}(x)}{u(x)}=-\frac{G^{\prime}(x)}{G(x)}$

where $G(x)=1-F(x)^{n}$. Notice that the right hand side of (4) is zero at $x=0$ as $G^{\prime}(0)=-n F(0)^{n-1} f(0)=0$ and $G(0)=1-F(0)^{n}=1$, whereas the left hand side is always positive. This means that $x=0$ cannot be a maximum.

Proof of Proposition 5. The expected utility of a scientist if the epistemic value is $x$ is given by

$$
E \hat{u}_{2}(x)=\left(F(x)^{n-1}-F(x)^{n}\right) u(x) .
$$

First order conditions for a maximum are now given by

$$
\begin{aligned}
& \left((n-1) F(x)^{n-2}-n F(x)^{n-1}\right) f(x) u(x)+u^{\prime}(x)\left(F(x)^{n-1}-F(x)^{n}\right)=0 . \\
& \text { As } f(x), u(x), u^{\prime}(x)>0 \text { and } F(x)^{n-1}-F(x)^{n}>0 \text { if } x>0, \text { this implies } \\
& (n-1) F(x)^{n-2}-n F(x)^{n-1}<0,
\end{aligned}
$$


or

(5) $1-F(x)<\frac{1}{n}$.

See that condition (5) implies $\hat{x}_{2}>x_{2}^{*}$.

Example 2. Showing no relation between $\hat{x}_{1}$ and $\hat{x}_{2}$ :

Take the utility function $u(x)=u+x^{\frac{1}{2}}$, and the uniform distribution function $F(x)=x$ when $x \in[0,1]$ and $F(x)=1$ when $x>1$. When $u \rightarrow 0$, the value $\hat{x}_{1}$ is calculated maximizing

$$
E \hat{u}_{1}(x)=\frac{x^{\frac{1}{2}}}{n}\left(1-x^{n}\right),
$$

with first order conditions given by

$$
x^{\frac{1}{2}}=\left(n+\frac{1}{2}\right) x^{n-\frac{1}{2}}
$$

and solution $\hat{x}_{1}=\left(\frac{1}{2 n+1}\right)^{\frac{1}{n}}$.

Under the inference rule $I R_{2}$, the optimal epistemic value maximizes

$$
E \hat{u}_{2}(x)=\left(x^{n-1}-x^{n}\right) x^{\frac{1}{2}}
$$

whose first order conditions are

$$
\left(n-\frac{1}{2}\right) x^{n-\frac{3}{2}}=\left(n+\frac{1}{2}\right) x^{n-\frac{1}{2}},
$$

resulting in $\hat{x}_{2}=\left(\frac{2 n-1}{2 n+1}\right)^{2}$.

When $n=3, \hat{x}_{1}=0,522>\hat{x}_{2}=0,51$, whereas when $n=4, \hat{x}_{1}=0,577<$ $\hat{x}_{2}=0,604$. By continuity, for small values of $u$ the same inequalities will hold.

Proof of Proposition 6. The expected utility of a scientist if the epistemic value is $x$ is given by

$$
E \hat{u}_{3}(x)=(1-F(x)) F(x)^{n-1} u(x)+(1-F(x))\left(1-F(x)^{n-1}\right) v(x)
$$

First order conditions for a maximum are given by

$$
\begin{aligned}
((n- & \left.1) F(x)^{n-2}-n F(x)^{n-1}\right) f(x) u(x)+(1-F(x)) F(x)^{n-1} u^{\prime}(x) \\
& +\left(n F(x)^{n-1}-(n-1) F(x)^{n-2}-1\right) f(x) v(x) \\
& +(1-F(x))\left(1-F(x)^{n-1}\right) v^{\prime}(x) \\
= & \left((n-1) F(x)^{n-2}(u(x)-v(x))-n F(x)^{n-1}(u(x)-v(x))-v(x)\right) f(x) \\
& +(1-F(x)) F(x)^{n-1} u^{\prime}(x)+(1-F(x))\left(1-F(x)^{n-1}\right) v^{\prime}(x) \\
= & 0,
\end{aligned}
$$


which gives

(6) $(n-1) F(x)^{n-2}-n F(x)^{n-1}=\frac{v(x)}{u(x)-v(x)}+H$,

where

$$
H=-\frac{(1-F(x))\left(F(x)^{n-1} u^{\prime}(x)+\left(1-F(x)^{n-1}\right) v^{\prime}(x)\right)}{f(x)}<0 .
$$

Equation (6) can be rewritten as

(7) $F(x)=\frac{n-1}{n}-\frac{v(x)}{(u(x)-v(x)) n F(x)^{n-2}}-\frac{H}{n F(x)^{n-2}}$.

When the maximum is attained at a corner solution, we have $\hat{x}_{3}=$ $x_{3}^{*}=0$. Otherwise we can compare equations (3) and (7) to show that $\hat{x}_{3}>x_{3}^{*}$. To this end notice first that $F(0)=0, F(x>\bar{x})=1,1>\frac{n-1}{n}>$ $\frac{n-1}{n}-\frac{v}{(u-v) n F(x>\bar{x})^{n-2}}$, and that $\frac{n-1}{n}-\frac{v}{(u-v)_{n}(x)^{n-2}}$ goes to $-\infty$ as $x$ goes to 0 . The maximizer $x_{3}^{*}$ is given by the intersection of curves $F(x)$ and $\frac{n-1}{n}-\frac{v}{(u-v) n F(x)^{n-2}}$. Further, the second order conditions require that the slope of $F(x)$ be greater that the slope of $\frac{n-1}{n}-\frac{v}{(u-v) n F(x)^{n-2}}$.

The right hand side of equation (7), $\frac{n-1}{n}-\frac{v(x)}{\left(u(x)-v(x) n F(x)^{n-2}\right.}-\frac{H}{n F(x)^{n-2}}=$ $\frac{n-1}{n}-\frac{v}{(u-v) n F(x)^{n-2}}$ at $\bar{x}$ and also goes to $-\infty$ as $x$ goes to 0 . However, for all values $x \in(0, \bar{x})$, we have that

$$
\begin{aligned}
& \frac{n-1}{n}-\frac{v(x)}{(u(x)-v(x)) n F(x)^{n-2}}-\frac{H}{n F(x)^{n-2}} \\
& >\frac{n-1}{n}-\frac{v}{(u-v) n F(x)^{n-2}} .
\end{aligned}
$$

Second order conditions for $\hat{x}_{3}$ to be a maximum require that the slope of $F(x)$ be greater than the slope $\frac{n-1}{n}-\frac{v(x)}{(u(x)-v(x)) n F(x)^{n-2}}-\frac{H}{n F(x)^{n-2}}$. This, together with the uniqueness of the maximum and inequality (8) imply that $\hat{x}_{3}>x_{3}^{*}$ (see Figure 1).

Proof of Proposition 7. Straightforward.

Proof of Proposition 8. Consider an inference rule $I R_{i}$ and a strategy vector $\left(x^{1}, \ldots, x^{n}\right)$ such that $f\left(x^{1}, \ldots, x^{n}\right)=x \neq x^{*}$, where $x^{*}$ is the optimal epistemic value for rule $I R_{i}$. By $\mathrm{U}$ there exists a scientist choosing $x^{i} \neq x^{*}$. If this scientist deviates to $x^{*}$ we have $f\left(x^{i-1},\left(x^{*}\right)^{i}\right)=x^{\prime}$, with $\left|x^{\prime}-x^{*}\right|<$ $\left|x-x^{*}\right|$. By IOD and the fact that the expected utility function has unique extreme points in the interval $[0, \bar{x}]$, the latter inequality implies a higher utility except if $x>\bar{x}$. 


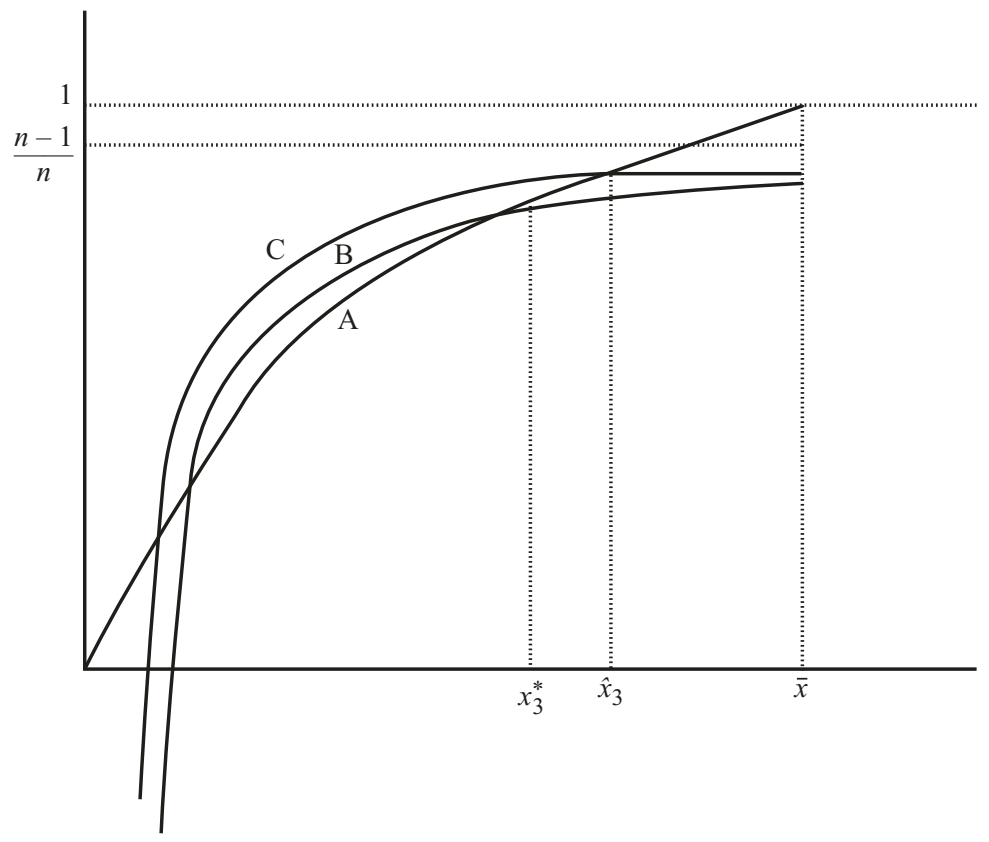

$$
\begin{gathered}
\mathrm{A} \text { is } F(x) \\
\mathrm{B} \text { is } \frac{n-1}{n}-\frac{v}{(u-v) n F^{n-2}} \\
\mathrm{C} \text { is } \frac{n-1}{n}-\frac{v(x)}{(u(x)-v(x)) n F^{n-2}}-\frac{H}{n F^{n-2}}
\end{gathered}
$$

FIGURE 1

\section{REFERENCES}

Aumann, R. 1959. Acceptable points in general cooperative n-person games. In Contributions to theory of games IV. Princeton University Press

Bernheim, B. D., B. Peleg and M. D. Whinston 1987. Coalition-proof Nash equilibria I: Concepts. Journal of Economic Theory 42:1-12

Bloor, D. 1976. Knowledge and social imaginery. Routledge and Kegan Paul

Brock, W. A. and S. N. Durlauf 1999. A formal model of theory choice in science, Economic Theory 14:113-30

Callon, M. 1994. Is science a public good? Science, Technology and Human Values 19:393-424

Cartwright, N. 1983. How the laws of physics lie. Clarendon Press

Cole, S. 1992. Making science: between nature and society. Harvard University Press

Gillies, D. 2000. Philosophical theories of probability. Routledge

Goldman, A. I. and M. Shaked. 1991. An economic model of scientific activity and truth acquisition, Philosophical Studies 63:31-55

Hull, D. L. 1988. Science as a process. University of Chicago Press

Kitcher, P. 1993. The advancement of science. Oxford University Press

Knorr-Cetina, K. D. 1981. The manufacture of knowledge. Oxford University Press

Kuhn, T. S. 1970. The structure of scientific revolutions. University of Chicago Press 
Kuhn, T. S. 1977. Objectivity, value judgments and theory choice. In The essential tension. University of Chicago Press

Latour, B. 1987. Science in action: how to follow scientists and engineers through society. Open University Press

Mäki, U. 1999. Science as a free market: a reflexivity test in an economics of economics. Perspectives on Science 74:486-509

Polany, M. 1962. The republic of science: its political and economic theory. Minerva 1:54-73

Popper, K. R. 1959. The logic of scientific discovery. Hutchinson

Radnitzky, G. 1987. The economic approach to the philosophy of science. British Journal for the Philosophy of Science 38:159-79

Wible, J. R. 1998. The economics of science: methodology and epistemology as if economics really mattered. Routledge

Zamora-Bonilla, J. P. 1999. Verisimilitude and the scientific strategy of economic theory. Journal of Economic Methodology 6:331-350

Zamora-Bonilla, J. P. 2002. Scientific inference and the pursuit of fame. Philosophy of Science 69:300-23 